\title{
INFLUÊNCIA DO EXERCÍCIO FÍSICO NA COGNIÇÃO: UMA ATUALIZAÇÃO SOBRE MECANISMOS FISIOLÓGICOS
}

\author{
INFLUENCE OF PHYSICAL EXERCISE ON COGNITION: AN UPDATE ON \\ PHYSIOLOGICAL MECHANISMS \\ INFLUENCIA DEL EJERCICIO FÍSICO EN LA COGNICIÓN: UNA ACTUALIZACIÓN \\ SOBRE MECANISMOS FISIOLÓGICOS
}

Artigo de Atualização

\begin{abstract}
Carlos Alberto Abujabra Merege
Filho ${ }^{1,2}$ (Professor de Educação Física)

Christiano Robles Rodrigues Alves ${ }^{2}$

(Professor de Educação Física)

Carlos Andrés Sepúlveda ${ }^{3}$

(Professor de Educação Física)

André dos Santos Costa ${ }^{4}$

(Professor de Educação Física)

Antônio Herbert Lancha Junior ${ }^{2}$

(Professor de Educação Física)

Bruno Gualano 1,2

(Professor de Educação Física)

1. Faculdade de Medicina,

Universidade de São Paulo, São

Paulo,SP, Brasil.

2. Escola de Educação Física e

Esporte, Universidade de São Paulo,

São Paulo, SP, Brasil.

3. Universidad Andrés Bello,

Facultad de Ciencias Biológicas,

Santiago, Chile.

4. Centro de Ciências da Saúde,

Universidade Federal de

Pernambuco, Recife, PE, Brasil.
\end{abstract}

\section{Correspondência:}

Bruno Gualano. Av. Prof. Mello

Moraes, 65, 05508-030, São Paulo,

SP, Brasil.

gualano@usp.br

\section{RESUMO}

Embora um crescente corpo de literatura corrobore o papel benéfico do exercício sobre a cognição, não há consenso sobre os mecanismos que norteiam as adaptações cerebrais agudas e crônicas ao exercício. A presente revisão narrativa tem como objetivo apresentar e discutir os mecanismos pelos quais o exercício afeta o desempenho cognitivo. Agudamente, especula-se que os efeitos do exercício sobre a resposta cognitiva sejam mediados por aumentos no fluxo sanguíneo cerebral e, por conseguinte, no aporte de nutrientes, ou por um aumento na atividade de neurotransmissores. Cronicamente, especula-se que o exercício possa promover adaptações em estruturas cerebrais e plasticidade sináptica que culminariam com melhoras cognitivas. Tais hipóteses são discutidas à luz das evidências científicas disponíveis, tanto em modelos animais quanto em humanos.

Palavras-chave: cérebro, atividade física, cognição.

\section{ABSTRACT}

Although a growing body of literature has supported the beneficial role of exercise on cognition, there is no consensus on the mechanisms underlying acute and chronic cerebral adaptations to exercise. The present review aims to present and discuss the mechanisms by which exercise affects cognitive performance. It has been speculated that the acute effects of exercise on cognitive response may be mediated by increases in cerebral blood flow and, hence, in nutrient availability, or by increases in neurotransmitter activity. It has been also postulated that chronic exercise may induce adaptations in brain structures and the synaptic plasticity, which would result in cognitive improvements. These hypotheses are discussed in light of available scientific evidence in animal models and humans

Keywords: brain, physical activity, cognition.

\section{RESUMEN}

Aunque un creciente cuerpo de literatura corrobore el papel benéfico del ejercicio sobre la cognición, no hay consenso sobre los mecanismos que nortean las adaptaciones cerebrales agudas y crónicas al ejercicio. La presente revisión narrativa tiene como objetivo presentar y discutir los mecanismos por los cuales el ejercicio afecta el desempeño cognitivo. Agudamente, se especula que los efectos del ejercicio sobre la respuesta cognitiva sean mediados por aumentos en el flujo sanguíneo cerebral y, por consiguiente, en el aporte de nutrientes, o por un aumento en la actividad de neurotransmisores. Crónicamente, se especula que el ejercicio pueda promover adaptaciones en estructuras cerebrales y plasticidad sináptica que culminarían con mejoras cognitivas. Tales hipótesis son discutidas a la luz de las evidencias científicas disponibles, tanto en modelos animales como en humanos.

Palabras clave: cerebro, actividad física, cognición.

\section{INTRODUÇÃO}

Há décadas, tem sido demonstrada uma relação benéfica entre a prática de exercícios físicos e o metabolismo do sistema nervoso central ${ }^{1,2}$. Interessantemente, logo após uma única sessão de exercício físico aeróbio realizado em intensidade moderada (i.e. $50 \%$ do $V_{2}{ }_{2 p i c o}$ ), são observadas melhoras no desempenho de diferentes tarefas cognitivas, tais como velocidade de processamento, atenção seletiva e memória de curto prazo (para revisão, ver ${ }^{3,4}$ ). Similarmente, recentes achados do nosso grupo e outros indicam que os exercícios de força e intermitentes (i.e. que combinam esforços de alta intensidade a períodos de repouso) também exercem uma influência positiva sobre a cognição ${ }^{5}$.

Sabidamente, indivíduos que praticam regularmente exercícios físicos apresentam melhor desempenho cognitivo quando comparados a seus pares sedentários ${ }^{2,6}$. Diante disso, cresce o interesse clínico e científico na aplicação do treinamento físico em diversas populações. Por exemplo, tem-se demonstrado que escolares fisicamente ativos apresentam uma maior facilidade no processo de aprendizagem, razão pela qual se sugere que o treinamento físico possa ser de suma 
relevância não apenas para o crescimento e desenvolvimento físico, mas também intelectual7. Além disso, há evidências de que a prática habitual de atividade física está associada a uma melhor função executiva em crianças com hiperatividade e déficit de atenção ${ }^{8}$. Sabe-se, ainda, que o treinamento físico pode abrandar a perda cognitiva na população idosa que, geralmente, apresenta piora nas capacidades desde processamento e manipulação de novas informações, baixo desempenho na realização de múltiplas tarefas simultaneamente, déficits de atenção e perda de memória de curta e longa duração ${ }^{9-12}$. Por fim, há evidências de que o treinamento físico possa atenuar os déficits cognitivos em pacientes acometidos por doenças neurodegenerativas ${ }^{12}$.

A despeito do crescente número de estudos demonstrando o papel do exercício físico - aguda ou cronicamente - sobre o desempenho cognitivo, os mecanismos que norteiam tais adaptações permanecem controversos e pouco explorados. Portanto, esta breve revisão narrativa tem como objetivo compilar e discutir as principais hipóteses fisiológicas que, potencialmente, explicariam os efeitos agudos e crônicos do exercício sobre a cognição.

Hipótese do aumento no fluxo sanguíneo regional e aporte energético: 0 cérebro, embora constitua apenas $2 \%$ da massa corporal, pode ser responsável por até 20\% do consumo energético total. Dessa forma, um substancial turnover de adenosina-trifosfato (ATP) faz se necessário para manter os potenciais de membrana, bem como as atividades de sinalização centrais e periféricas ${ }^{13}$.

Nesse contexto, o fornecimento de oxigênio e nutrientes através do fluxo sanguíneo cerebral é determinante para suprir tamanha demanda energética ${ }^{13,14}$. Em repouso, o fluxo sanguíneo cerebral gira em torno de 15\% do débito cardíaco total ( $750 \mathrm{~mL} / \mathrm{min})$. O controle fino deste fluxo é efetuado por mecanismos específicos de autorregulação, sendo pouco influenciado pelo sistema nervoso autônomo ${ }^{14-16}$. Entretanto, eventualmente, pode ocorrer uma redistribuição desse fluxo a fim de suprir a demanda energética de áreas cerebrais em pleno funcionamento ${ }^{15}$. De fato, é bem estabelecido que uma atividade motora dinâmica eleva a demanda energética em áreas do córtex responsáveis pelo controle motor, tais como área pré-motora, motora suplementar e sensoriomotora ${ }^{15}$. Portanto, é possível especular que essa redistribuição do fluxo esteja relacionada ao melhor desempenho em tarefas cognitivas.

Corroborando tal hipótese, após uma sessão aguda de exercício físico, parece ocorrer um aumento significativo no fluxo sanguíneo cerebral ${ }^{15,17}$. Por exemplo, Smith et al. ${ }^{17}$ avaliaram o fluxo sanguíneo cerebral por meio de uma técnica de ressonância magnética (arterial spin labeling), antes e imediatamente após uma sessão de exercício físico em cicloergômetro em intensidade moderada (escore 13 de percepção subjetiva de esforço na escala 6-20 de Borg). Os achados indicaram um aumento significativo no fluxo sanguíneo cerebral logo após a sessão aguda de exercício físico (+20\%). Tendo em vista que maior fluxo de sangue no cérebro (ou em suas diferentes regiões) representaria maior oferta de oxigênio e nutrientes (i.e. carboidratos, creatina) e, por conseguinte, maior aporte energético (i.e. ATP) pode-se assumir que esse seja um provável mecanismo através do qual o exercício agudo favorece o desempenho cognitivo ${ }^{14-18}$.

Hipótese de maior atividade de neurotransmissores: Há estudos que indicam que o exercício físico agudo é capaz de elevar a síntese de neurotransmissores sinápticos ${ }^{19,22}$. Por exemplo, Sharma et al. ${ }^{21}$ demonstraram que o exercício físico prolongado de intensidade moderada atua na ativação das catecolaminas cerebrais, facilitando sua entrada através da barreira hematoencefálica, provavelmente em função da elevação na temperatura corporal induzida pelo exercício. Em suporte a essa hipótese, Watson et al..$^{22}$ submeteram um grupo de sujeitos a condições de temperatura diferentes $\left(39^{\circ} \mathrm{C}\right.$ e $\left.35^{\circ} \mathrm{C}\right)$ e encontraram diferenças significativas nas concentrações de $\mathbf{S 1 0 0 \beta}$ (maior a $\left.3^{\circ} \mathrm{C}\right)$, uma proteína especifica do metabolismo cerebral utilizada como marcador sanguíneo de rompimento da permeabilidade da barreira hematoencefálica. Especula-se que, em consequência de um aumento na permeabilidade da barreira hematoencefálica, as catecolaminas podem ingressar mais facilmente no sistema nervoso central. De fato, sabe-se que a sensação de bem-estar experimentada após uma sessão de exercício físico realizado em intensidade moderada parece estar diretamente associada a maior ação de neurotransmissores, tais como: noradrenalina, $\beta$-endorfina e a própria dopamina ${ }^{23-26}$.

O papel da intensidade do exercício agudo sobre a resposta cognitiva: Embora seja bem aceito que uma única sessão de exercício físico possa aumentar substancialmente o desempenho cognitivo, Chmura et al. ${ }^{19}$ encontram efeitos negativos sobre a cognição. A intensidade na qual o exercício físico é realizado parece explicar tal contradição 20 . Nesse sentido, tem sido proposta uma relação em "U invertido" entre a ativação do sistema nervoso central e a intensidade com a qual o exercício é realizado ${ }^{19,20}$. Sendo assim, uma sessão de exercício físico aeróbio realizado de forma contínua em intensidade moderada $\left(\sim 60 \% \mathrm{VO}_{2 \text { máx }}\right)$ poderia promover um efeito benéfico em importantes funções cognitivas, tais como velocidade de processamento, atenção seletiva e controle inibitório ${ }^{27,28}$. Em contrapartida, uma sessão aguda de exercício físico contínuo realizado em alta intensidade $\left(\sim 80 \% \mathrm{VO}_{2}\right.$ máx) exerceria efeitos prejudiciais sobre a cognição ${ }^{18-20}$. Na tentativa de explicar tal relação entre intensidade de exercício e resposta cognitiva, Tomporowski ${ }^{29}$ sugere que o exercício físico em alta intensidade poderia provocar um quadro de fadiga sistêmica, resultando tanto na queda do desempenho físico quanto cognitivo. Interessantemente, recentes achados do nosso grupo demonstram que uma única sessão de exercício físico em alta intensidade realizada de maneira intervalada (i.e. dez sprints de um minuto de duração em intensidade correspondente a 80\% FC máxima, intercalados por pausas ativas de um minuto em intensidade correspondente a $60 \%$ da FC máxima) não prejudicou a memória de curto prazo e até mesmo melhorou a velocidade de processamento (avaliada pelo Stroop Test) ${ }^{5}$. Esses dados sugerem que as pausas ativas realizadas entre os esforços possam ter minimizado o quadro de fadiga, evitando assim os prejuízos na cognição. Especula-se que a redução de desempenho cognitivo acarretada pela fadiga possa estar associada à redução do aporte sanguíneo e energético cerebral bem como à supressão da síntese de neurotransmissores ${ }^{30-32}$. De certa forma, essa possibilidade é sustentada pelas duas hipóteses anteriores que se propõem a explicar mecanismos pelos quais o exercício agudo atua sobre o desempenho cognitivo.

Hipótese de adaptações em estruturas cerebrais e plasticidade sináptica: Os avanços das técnicas não invasivas de neuroimagem, como a espectroscopia por ressonância magnética e o eletroencefalograma, vêm colaborando substancialmente para o entendimento dos mecanismos envolvidos na positiva relação entre a prática de exercícios físicos e o desempenho em tarefas cognitivas ${ }^{2}$. De fato, diversos estudos têm revelado que indivíduos fisicamente ativos apresentam maiores ativações no espectro de bandas específicas (i.e.theta, alpha e beta) no exame de eletroencefalograma quando comparados a seus pares sedentários, o que sugere padrão de ativação cerebral diferenciado em função do nível individual de atividade física ou capacidade física ${ }^{33-35}$. Além disso, jovens fisicamente ativos parecem apresentar maior ativação em áreas cerebrais específicas, sugerindo a existência de uma maior rede de estruturas neurais em diversas regiões cerebrais, tais como lóbulo frontal, córtex cingulado anterior, lóbulo infra-temporal e córtex parietal, em decorrência da atividade física regular. Curiosamente, cada uma dessas regiões está envolvida na realização de tarefas cognitivas específicas².

Estudos que utilizaram o exame de espectroscopia por ressonância 
Tabela 1. Efeito agudo do exercício no metabolismo cerebral em modelos animais e humanos.

\begin{tabular}{|c|c|c|c|c|}
\hline Autor & Amostra & $\begin{array}{l}\text { Hipótese testada e técnica } \\
\text { utilizada }\end{array}$ & $\begin{array}{l}\text { Tipo, intensidade e duração do } \\
\text { exercício físico. }\end{array}$ & Principais achados \\
\hline $\begin{array}{l}\text { Sharma } \\
\text { et al. }(1991)^{21}\end{array}$ & 96 roedores & $\begin{array}{l}\text { Aumento na permeabilidade da } \\
\text { barreira hematoencefálica e } \\
\text { aumento na concentração de } \\
\text { neurotransmissores - Evans Blue e } \\
{ }^{131} \text {-sódio e atividade serotoninérgica } \\
\text { avaliada por p-clorofenilanina. }\end{array}$ & $\begin{array}{l}\text { Aeróbio em meio aquático } \\
\text { durante } 30 \text { minutos. }\end{array}$ & $\begin{array}{c}\text { Após } 30 \text { minutos de exercício } \\
\text { houve um aumento médio de } 600 \% \text { na } \\
\text { permeabilidade da barreira } \\
\text { hematoencefálica e } 148,4 \% \text { na } \\
\text { concentração plasmática e } \\
\text { cerebral de serotonina. }\end{array}$ \\
\hline $\begin{array}{l}\text { Ogoh } \\
\text { et al. }(2005)^{32}\end{array}$ & $\begin{array}{l}7 \text { homens } \\
\text { (idade: } 23,0 \pm 2,0 \text { anos) }\end{array}$ & $\begin{array}{c}\text { Alteração do fluxo sanguíneo } \\
\text { cerebral - relação da velocidade do } \\
\text { fluxo sanguíneo cerebral na artéria } \\
\text { braquial e na veia jugular } \\
\text { mensurado por cateterismo. }\end{array}$ & $\begin{array}{c}\text { Aeróbio contínuo em cicloergômetro. } \\
\text { A carga inicial foi correspondente a } \\
\text { frequência cardíaca de } 160 \text { bpm. Essa } \\
\text { carga foi mantida até a exaustão } \\
\text { mesmo quando a frequência } \\
\text { cardíaca aumentou. }\end{array}$ & $\begin{array}{c}\text { Houve um aumento médio de 18,6\% } \\
\text { no fluxo sanguíneo cerebral nos } \\
\text { primeiros cinco minutos de exercício. } \\
\text { Não houve alteração no fluxo sanguíneo } \\
\text { cerebral no momento da exaustão em } \\
\text { relação ao repouso. }\end{array}$ \\
\hline $\begin{array}{l}\text { Bhambhani } \\
\text { et al. }(2007)^{31}\end{array}$ & $\begin{array}{c}17 \text { homens } \\
\text { (idade: } 26.7 \pm 8.6 \text { anos) }\end{array}$ & $\begin{array}{c}\text { Alteração no fluxo sanguíneo } \\
\text { cerebral - espectroscopia } \\
\text { por infravermelho }\end{array}$ & $\begin{array}{c}\text { Aeróbio em cicloergômetro com } \\
\text { incremento de carga (30 W) a cada } 2 \\
\text { minutos até atingir o limiar anaeróbio. }\end{array}$ & $\begin{array}{l}\text { Após o limiar anaeróbio houve um declinio } \\
\text { médio de } 75 \% \text { na oxigenação cerebral. }\end{array}$ \\
\hline $\begin{array}{l}\text { Ando } \\
\text { et al. }(2011)^{18}\end{array}$ & $\begin{array}{l}12 \text { homens } \\
\text { (idade: } 25,3 \pm 3,1 \text { anos) }\end{array}$ & $\begin{array}{l}\text { Alteração no fluxo sanguíneo } \\
\text { cerebral - espectroscopia } \\
\text { por infravermelho } \\
\text { (Oxi-hemoglobina/ } \\
\text { deoxi-hemoglobina). }\end{array}$ & $\begin{array}{l}\text { Aeróbio em esteira durante } 18 \text { minutos } \\
\text { com incremento de carga a cada } 6 \\
\text { minutos para atingir a cada uma das } \\
\text { seguintes intensidades: } 40 \%, 60 \% \text { e } \\
80 \% \text { do } \mathrm{VO}_{2 \text { máx. }}\end{array}$ & $\begin{array}{l}\text { Diminuição de aproximadamente } 3,4 \% \text { no } \\
\text { fluxo sanguíneo cerebral em intensidade } \\
\text { correspondente a } 80 \% \text { do } \mathrm{VO}_{2 \text { máx. }}\end{array}$ \\
\hline $\begin{array}{l}\text { Hasegawa } \\
\text { et al. }(2011)^{26}\end{array}$ & 11 roedores & $\begin{array}{c}\text { Aumento na atividade de } \\
\text { neurotransmissores - microdiálise, } \\
\text { biotelemetria e } \\
\text { monitoramento metabólico. }\end{array}$ & $\begin{array}{l}\text { Aeróbio em esteira rolante à } 26 \text { m/min } \\
\text { (60\% consumo máximo de oxigênio) } \\
\text { (duração total de } 80 \text { minutos). }\end{array}$ & $\begin{array}{c}\text { Aumento médio nos níveis plasmáticos de } \\
\text { noradrenalina (500\%) e dopamina ( } 833 \%) \\
\text { durante o período de treinamento. Não } \\
\text { houve alteração nos níveis plasmáticos de } \\
\text { serotonina. }\end{array}$ \\
\hline $\begin{array}{l}\text { Goekint } \\
\text { et al. }(2012)^{26}\end{array}$ & 7 roedores & $\begin{array}{l}\text { Aumento na atividade de } \\
\text { neurotransmissores - microdiálise } \\
\text { e monitoramento metabólico. }\end{array}$ & $\begin{array}{l}\text { Exercício em esteira rolante a } 20 \mathrm{~m} / \\
\text { min ( } 60 \text { minutos). }\end{array}$ & $\begin{array}{l}\text { Aumento médio de } 110,0 \% \text { no conteúdo } \\
\text { de dopamina hipocampal. }\end{array}$ \\
\hline
\end{tabular}

Tabela 2. Efeitos do exercício físico crônico no metabolismo cerebral em modelos animais e humanos.

\begin{tabular}{|c|c|c|c|c|}
\hline Autor & Amostra & $\begin{array}{c}\text { Hipótese testada e técnica } \\
\text { utilizada }\end{array}$ & $\begin{array}{l}\text { Tipo, intensidade e duração do } \\
\text { exercício físico. }\end{array}$ & Principais achados \\
\hline $\begin{array}{l}\text { Dustman } \\
\text { et al. }(1990)^{34}\end{array}$ & $\begin{array}{l}60 \text { homens } \\
\text { (adultos de } 20-30 \text { anos } \\
\text { e idosos de } 50-62 \text { anos). }\end{array}$ & $\begin{array}{l}\text { Maior nível de aptidão física } \\
\text { correlacionada com melhor } \\
\text { ativação cerebral - } \\
\text { eletroencefalograma. }\end{array}$ & $\begin{array}{l}\text { Nível de aptidão física foi estimado por } \\
\text { teste de consumo máximo } \\
\text { de oxigênio }\left(\mathrm{VO}_{2 \text { máx }}\right) \text {. }\end{array}$ & $\begin{array}{l}\text { Adultos e idosos com maior aptidão física } \\
\text { demonstraram maior ativação cerebral em } \\
\text { comparação a seus pares de menor aptidão } \\
(+3,5 \% \text { e } 2,5 \% \text {, respectivamente). }\end{array}$ \\
\hline $\begin{array}{l}\text { Lardon e Polish } \\
\qquad(1996)^{35}\end{array}$ & $\begin{array}{l}24 \text { homens e } \\
12 \text { mulheres (31 anos). }\end{array}$ & $\begin{array}{l}\text { Maior nível de aptidão física } \\
\text { correlacionada com melhor ativação } \\
\text { cerebral - eletroencefalograma. }\end{array}$ & $\begin{array}{c}\text { Nível de atividade física foi estimado a } \\
\text { partir do total de horas participadas } \\
\text { em atividades esportivas } \\
\text { (total de horas por semana). }\end{array}$ & $\begin{array}{l}\text { Individuos com maior nível de atividade } \\
\text { física desmonstraram maiores ativações na } \\
\text { frequencia média das ondas theta, alpha e } \\
\text { beta }(+175 \%) \text { do córtex cerebral. }\end{array}$ \\
\hline $\begin{array}{l}\text { Van Praag } \\
\text { et al. }(1999)^{42}\end{array}$ & $\begin{array}{c}33 \text { roedores } \\
\text { (15 adultos e } 18 \text { velhos) }\end{array}$ & $\begin{array}{l}\text { Neurogênese - imunohistoquimica } \\
\text { por imunofluorescência e } \\
\text { aprendizagem espacial. }\end{array}$ & $\begin{array}{l}\text { A atividade física foi administrada pela } \\
\text { roda de atividade espontânea } \\
\text { com acesso ilimitado } \\
\text { (média de } 4.9 \mathrm{~km} \text { percorridos). }\end{array}$ & $\begin{array}{l}\text { Aumento de aproximadamente } 55,6 \% \text { na } \\
\text { quantidade de células neurais em } \\
\text { proliferação nos grupos treinados. }\end{array}$ \\
\hline $\begin{array}{l}\text { Colcombe et al. } \\
\qquad(2004)^{40} \\
\text { estudo } 1^{*}\end{array}$ & $\begin{array}{l}41 \text { idosos } \\
\text { (idade: } 67 \text { e } 68 \text { anos). }\end{array}$ & $\begin{array}{l}\text { Maior nível de aptidão física } \\
\text { correlacionada com melhor } \\
\text { ativação cerebral - } \\
\text { ressonância magnética. }\end{array}$ & $\begin{array}{l}\text { O nível de aptidão física foi avaliado } \\
\text { pelo consumo máximo de } \\
\text { oxigênio }\left(\mathrm{VO}_{2 \text { máx }}\right) \text {. }\end{array}$ & $\begin{array}{c}\text { Melhores resultados no teste cognitivo e } \\
\text { maior aumento na ativação cortical das } \\
\text { regiões 8, 32, } 40 \text { e 46, em idosos com maior } \\
\text { aptidão física. }\end{array}$ \\
\hline $\begin{array}{l}\text { Colcombe et al. } \\
\qquad(2004)^{40} \\
\text { estudo } 2^{*}\end{array}$ & $\begin{array}{c}18 \text { mulheres } \\
\text { e } \\
11 \text { homens } \\
\text { (idade: } 65,60 \pm 5,66 \\
\text { anos). }\end{array}$ & $\begin{array}{l}\text { Treinamento físico melhora a } \\
\text { ativação cerebral - ressonância } \\
\text { magnética e teste cognitivo de } \\
\text { descriminação de estímulos } \\
\text { (Flanker Task). }\end{array}$ & $\begin{array}{c}3 \text { sessões por semana de caminhada } \\
\left(\sim 40-70 \% \mathrm{FC}_{\text {máx }}\right) \text { durante um } \\
\text { período de } 6 \text { meses. }\end{array}$ & $\begin{array}{l}\text { Grupo treinando mostrou redução de } 11 \% \\
\text { no conflito de resposta na tarefa de } \\
\text { descriminação de estímulos e um aumento } \\
\text { ativação cerebral na área de } \\
\text { controle da atenção. }\end{array}$ \\
\hline $\begin{array}{l}\text { Van der Borght } \\
\text { et al. }(2009)^{52}\end{array}$ & 40 roedores & $\begin{array}{l}\text { Neurogênese e Angiogênese - } \\
\text { imunocitoquimica do hipocampo } \\
\text { (endotélio e neurônio). }\end{array}$ & $\begin{array}{l}\text { Exercício aeróbio em esteira rolante, } \\
\text { divididos em dois períodos. } 1^{\circ} \text { grupo: } \\
5 \text { a } 6 \text { km de distancia percorrida no } \\
\text { treinamento durante } 3 \text { dias. } 2^{\circ} \text { grupo: } \\
13 \mathrm{~km} \text { de distância percorrida no } \\
\text { treinamento durante } 10 \text { dias. }\end{array}$ & $\begin{array}{c}\text { Os } 3 \text { primeiros dias de treinamento } \\
\text { aumentou aproximadamente } 23,1 \% \text { a } \\
\text { quantidade do transportador de glicose } \\
\text { Glut-1,um marcador de densidade } \\
\text { endotelial. } 10 \text { dias de treinamento } \\
\text { aumentou aproximadamente } 60 \% \text { a prolifera- } \\
\text { ção de células neurais do giro dentado. }\end{array}$ \\
\hline
\end{tabular}

magnética corroboram a hipótese de que indivíduos treinados apresentam padrão diferenciado de ativação cerebral quando comparados a não treinados ${ }^{36-39}$. Colcombe et al. ${ }^{40}$ conduziram dois elegantes experimentos, nos quais demonstraram que indivíduos com alta aptidão aeróbia (experimento \#1) ou indivíduos submetidos a treinamento físico aeróbio (experimento \#2) apresentam maior ativação nas regiões corticais pré-frontal e parietal, quando comparados àqueles com baixa aptidão física e indivíduos controles não treinados, respectivamente.

A fim de investigar mecanismos biomoleculares envolvidos no remodelamento das estruturas cerebrais e na maior plasticidade sináptica, diversas pesquisas utilizaram de métodos invasivos em modelos animais. Destacam-se os estudos que demonstraram 1) proliferação de 
novos capilares cerebrais, 2) neurogênese e 3) surgimento de novas conexões sinápticas após a realização de treinamento aeróbio regu$\mid a r^{41-43}$. Estas alterações parecem ocorrer principalmente por conta de importantes ações hormonais. Enquanto alguns hormônios exercem funções de estímulo e crescimento neuronal, tais como Insuline Growth Factor I (IGF-I) e Vascular Endothelial Growth Factor (VEGF), outros podem desempenhar um papel deletério no sistema nervoso central, tal como o cortisol. Portanto, a compreensão acerca dos efeitos do treinamento físico sobre a produção hormonal faz-se relevante no entendimento da adaptação do sistema nervoso central ao treinamento.

O IGF-1 é um dos principais candidatos a explicar os benefícios do exercício crônico sobre o desempenho cognitivo. Trata-se de um hormônio que induz neurogênese, cujas concentrações séricas guardam relação direta com os resultados em testes de aprendizagem espacial em ratos ${ }^{44}$. Além disso, sabe-se que indivíduos com altas concentrações séricas de IGF-I apresentam baixas concentrações plasmáticas de homocisteína, um aminoácido sulfuroso derivado da demetilação da metionina e que, em altos níveis, pode causar lesões cerebrais e transtornos neuropsiquiátricos ${ }^{45}$. Além disso, a homocisteína é pró-aterogênica e pró-trombótica, elevando o risco de acidente vascular cerebral e neurotoxidade ${ }^{46}$. No hipocampo, o IGF-I também modula os níveis de acetilcolina, a qual exerce importantes funções no desenvolvimento do sistema nervoso central ${ }^{47}$. Interessantemente, Casilhas et al. ${ }^{10}$ observaram aumentos nas concentrações de IGF-1 em idosos submetidos a 24 semanas de treinamento de força e apresentaram meIhoras cognitivas (ex: memória, atenção), enfatizando o potencial papel desse hormônio nas adaptações cerebrais induzidas pelo exercício.

Outro hormônio que vem sendo estudado devido a seu papel ímpar na angiogênese cerebral é o VEGF ${ }^{48-51}$. Um elegante estudo de bloqueio farmacológico revelou que a angiogênese é um dos principais fatores responsáveis pela aquisição de memória e aprendizagem em ratos $^{49}$. Supostamente, tal achado se explica em razão da iminente necessidade de novos vasos sanguíneos, responsáveis por manterem a demanda energética nos processos de diferenciação, migração e posterior maturação neural48. É imperativo destacar que o treinamento físico é considerado um importante estimulador do VEGF ${ }^{50,51}$. Os efeitos positivos da atividade física na plasticidade cerebral e na função cognitiva podem, em parte, ser mediados por um estímulo salientado sobre a vascularização cerebral e o fluxo sanguíneo local ${ }^{52}$.

Sabe-se, ainda, que o eixo endócrino hipotalámico-hipofisário-adrenocortical é extremamente influenciado pelo treinamento físico. Estudos tanto em modelos animais quanto em humanos demonstram que o treinamento físico pode gerar adaptações nas glândulas adrenais, reduzindo a liberação de cortisol e corticosterona ${ }^{24,53}$. Sabidamente, elevações séricas de corticosterona inibem a diferenciação de células progenitoras no hipocampo ${ }^{53,54}$. Além disso, há boas evidências de que o excesso de corticosterona produz um comprometimento da função hipocampal, tal como a memória de trabalho e a espacial ${ }^{55,56}$. Evidentemente, estratégias que visem a redução de cortisol e corticosterona são de potencial valia nas adaptações cognitivas, de modo que o efeito supressor do exercício sobre tais hormônios merece destaque.

Por fim, cabe ressaltar que o treinamento físico regular de intensidade moderada exerce ação antioxidante ${ }^{57,58}$. Embora os marcadores de estresse oxidativo normalmente avaliados sejam plasmáticos ou séricos, é possível especular que essa ação antioxidante do exercício seja sistêmica e, como tal, atinja também o sistema nervoso central. Considerando que o estresse oxidativo exacerbado é um dos candidatos responsáveis pelo envelhecimento e, consequentemente, pelo declínio de diversas funções executivas, possivelmente a ação antioxidante do treinamento físico possa se constituir como um adicional mecanismo pelo qual o exercício melhora a cognição ${ }^{59}$. De fato, mais estudos randomizados com humanos devem ser conduzidos para comprovar ou não essa premissa.

\section{CONSIDERAÇÕES FINAIS}

Os benefícios agudos e crônicos do exercício físico sobre o desempenho cognitivo têm sido amplamente reportados. Agudamente, tais efeitos devem-se, provavelmente, a um aumento do fluxo sanguíneo cerebral e, por conseguinte, do aporte de nutrientes. Paralelamente, há evidências sugerindo que o efeito agudo do exercício possa ser mediado por um aumento na atividade de neurotransmissores. Cronicamente, especula-se que o exercício possa promover adaptações em estruturas cerebrais e na plasticidade sináptica que culminariam com melhoras cognitivas (figura 1). Muitas evidências acerca dos mecanismos que norteiam as positivas respostas cognitivas ante o exercício agudo e

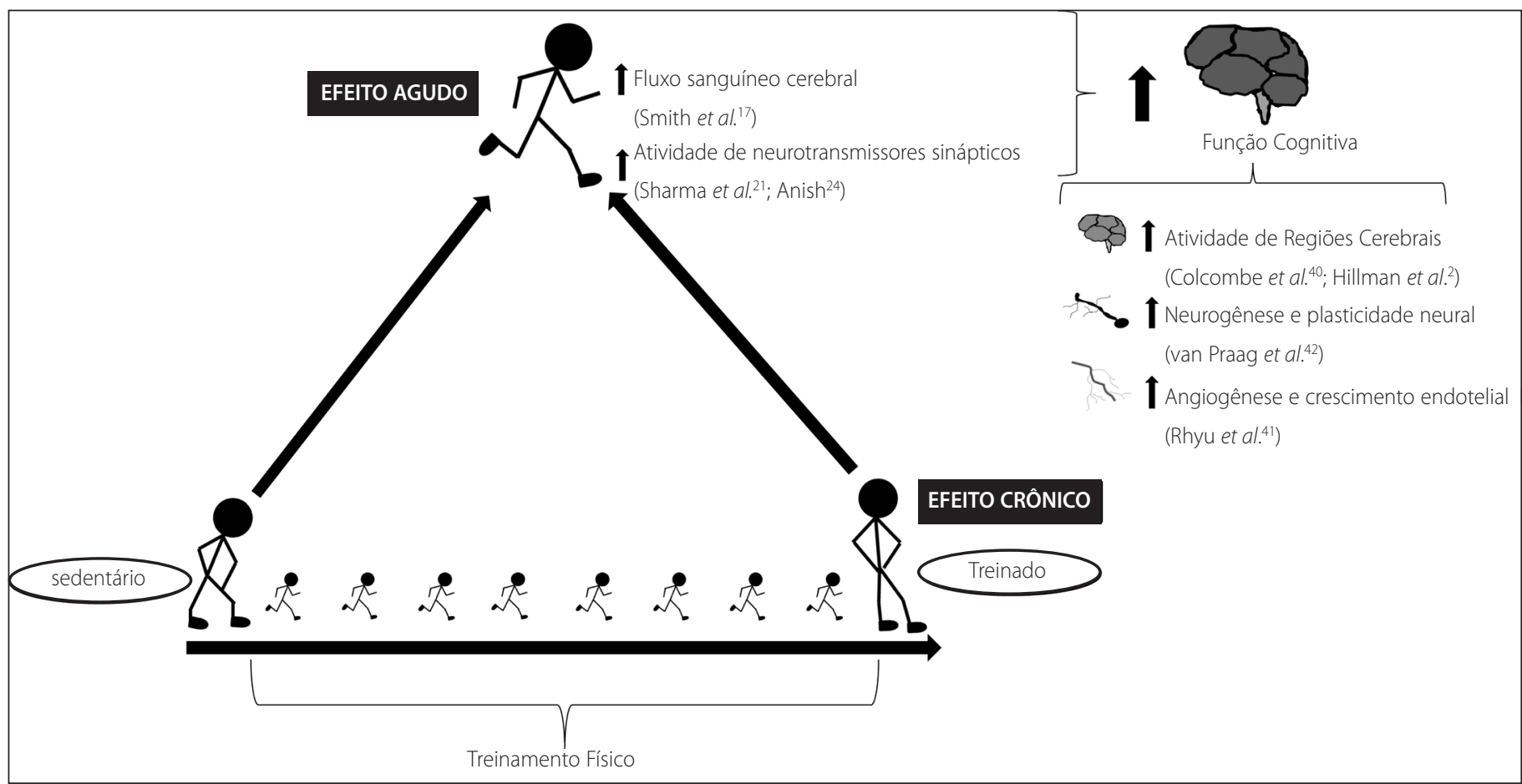

Figura 1. Mecanismos fisiológicos que explicariam o papel agudo e crônico do exercício sobre a cognição. 
crônico são produzidas em modelos animais. Em virtude dos avanços científicos e tecnológicos que têm permitido o desenvolvimento de novos e sofisticados métodos de imagem cerebral, tem-se aumentado a compreensão dos mecanismos pelos quais o exercício interfere na função cognitiva. Tais estudos mecanísticos são de grande relevância para a elaboração de modelos eficazes de treinamento objetivados a melhorar a cognição em populações saudáveis e também naquelas acometidas por demência.

\section{REFERÊNCIAS}

1. Spirduso WW. Physical fitness, aging, and psychomotor speed: a review. J Gerontol. 1980;35(6):850-65.

2. Hillman CH, Erickson KI, Kramer AF. Be smart, exercise your heart: exercise effects on brain and cognition. Nature Reviews Neuroscience. 2008;9(1):58-65.

3. McMorris T, Sproule J, Turner A, Hale BJ. Acute, intermediate intensity exercise, and speed and accuracy in working memory tasks: a meta-analytical comparison of effects. Physiol Behav. 2011;1;102(3-4):421-8.

4. Lambourne K, Tomporowski P. The effect of exercise-induced arousal on cognitive task performance: a meta-regression analysis. Brain Res. 2010;23;1341:12-24.

5. Alves CRR, Tessaro VH, Teixeira LAC, Murakava K, Roschel H, Gualano B, Takito MY. Influence of an acute high-intensity interval training session on selective attention and short-term memory tasks. Percept Mot Skills. 2014;118(1):63-72.

6. Etnier $J$, Chang YK. The effect of physical activity on executive function: a brief commentary on definitions, measurement issues, and the current state of the literature. J Sport Exerc Psychol. 2009:31(4):469-83

7. Hillman $\mathrm{CH}$, Castelli DM, Buck SM. Aerobic fitness and neurocognitive function in healthy preadolescent children. Med Sci Sports Exerc. 2005;37(11):1967-74.

8. Gapin J, Etnier JL. The Relationship Between Physical Activity and Executive Function Performance in Children With Attention-Deficit Hyperactivity Disorder. J Sport Exercise Psy. 2010;32(6):753-63.

9. Kramer AF, Erickson KI, Colcombe SJ. Exercise, cognition, and the aging brain. J Appl Physiol. 2006;101(4):1237-42.

10. Cassilhas RC, Viana VAR, Grassmann V, Santos RT, Santos RF, Tufik S, et al. The impact of resistance exercise on the cognitive function of the elderly. Med Sci Sport Exer. 2007;39(8):1401-7.

11. Spirduso WW, Francis K, Eakin T, Stanford C. Quantification of manual force control and tremor. J Mot Behav.2005;37(3):197-210

12. Karceski S. Preventing Alzheimer disease with exercise? Neurology. 2012;24;78(17):e110-2.

13. Andres RH, Ducraya AD, Schlattner U, Wallimann T, Widmer HR. Functions and effects of creatine in the central nervous system. Brain Res Bull. 20081;76(4):329-43.

14. Ide K, Secher NH. Cerebral blood flow and metabolism during exercise. Prog Neurobiol. 2000;61(4):397-414.

15. Ogoh S, Ainslie PN. Cerebral blood flow during exercise: mechanisms of regulation. J Appl Physiol. 2009;107(5):1370-80

16. Tamayo-Orrego L, Duque-Parra JE. [The metabolic regulation of cerebral microcirculation]. Rev Neurol. 2007;1-15;44(7):415-25

17. Smith JC, Paulson ES, Cook DB, Verber MD, Tian Q. Detecting changes in human cerebral blood flow after acute exercise using arterial spin labeling: implications for fMRI. J Neurosci Methods. 2010;30;191(2):258-62.

18. Ando S, Kokubu M, Yamada Y, Kimura M. Does cerebral oxygenation affect cognitive function during exercise? Eur J Appl Physiol. 2011;111(9):1973-82.

19. Chmura J, Nazar K, Kaciuba-Uscilko H. Choice reaction time during graded exercise in relation to blood lactate and plasma catecholamine thresholds. Int J Sports Med. 1994;15(4):172-6.

20. Kashihara K, Maruyama T, Murota M, Nakahara Y. Positive effects of acute and moderate physical exercise on cognitive function. J Physiol Anthropol. 2009;28(4):155-64.

21. Sharma HS, Cervos-Navarro J, Dey PK. Increased blood-brain barrier permeability following acute short-term swimming exercise in conscious normotensive young rats. Neurosci Res. 1991;10(3):211-21.

22. Watson P, Shirreffs SM, Maughan RJ. Blood-brain barrier integrity may be threatened by exercise in a warm environment. Am J Physiol-Reg I. 2005288(6):R1689-R94.

23. McMorris T, Tallon M, Williams C, Sproule J, Draper S, Swain J, et al. Incremental exercise, plasma concentrations of catecholamines, reaction time, and motor time during performance of a noncompatible choice response time task. Percept Motor Skill. 2003;97(2):590-604.

24. Anish EJ. Exercise and its effects on the central nervous system. Curr Sports Med Rep. 2005;4(1):18-23.

25. Hasegawa H, Takatsu S, Ishiwata T, Tanaka H, Sarre S, Meeusen R. Continuous monitoring of hypothalamic neurotransmitters and thermoregulatory responses in exercising rats. J Neurosci Meth. 2011;15;202(2):119-23.

26. Goekint M, Bos I, Heyman E, Meeusen R, Michotte Y, Sarre S. Acute running stimulates hippocampal dopaminergic neurotransmission in rats, but has no influence on brain-derived neurotrophic factor. J Appl Physiol. 2011;1.

27. Yanagisawa H, Dan I, Tsuzuki D, Kato M, Okamoto M, Kyutoku Y, et al. Acute moderate exercise elicits increased dorsolateral prefrontal activation and improves cognitive performance with Stroop test. Neuroimage. 2010;1;50(4):1702-10.

28. Hogervorst E, Riedel W, Jeukendrup A, Jolles J. Cognitive performance after strenuous physical exercise. Percept Mot Skills. 1996;83(2):479-88.

29. Tomporowski PD. Effects of acute bouts of exercise on cognition. Acta Psychol (Amst). 2003;112(3):297-324

30. Ando S, Yamada Y, Kokubu M. Reaction time to peripheral visual stimuli during exercise under hypoxia.

\section{AGRADECIMENTOS}

Conselho Nacional de Desenvolvimento Científico e Tecnológico (CNPq) e Coordenação de Aperfeiçoamento de Pessoal de Nível Superior (CAPES).

Todos os autores declararam não haver qualquer potencial conflito de interesses referente a este artigo.

J Appl Physiol. 2010;108(5):1210-6.

31. Bhambhani Y, Malik R, Mookerjee S. Cerebral oxygenation declines at exercise intensities above the respiratory compensation threshold. Respir Physiol Neurobiol. 2007;14;156(2):196-202.

32. Ogoh S, Dalsgaard MK, Yoshiga CC, Dawson EA, Keller DM, Raven PB, et al. Dynamic cerebral autoregulation during exhaustive exercise in humans. Am J Physiol Heart Circ Physiol. 2005;288(3):H1461-7.

33. Dustman RE, LaMarche JA, Cohn NB, Shearer DE, Talone JM. Power spectral analysis and cortical coupling of EEG for young and old normal adults. Neurobiol Aging. 1985;6(3):193-8.

34. Dustman RE, Emmerson RY, Ruhling RO, Shearer DE, Steinhaus LA, Johnson SC, et al. Age and fitness effects on EEG, ERPs, visual sensitivity, and cognition. Neurobiol Aging. 1990;11(3):193-200.

35. Lardon MT, Polich J. EEG changes from long-term physical exercise. Biol Psychol. 1996;27;44(1):19-30.

36. Kramer AF, Hahn S, Cohen NJ, Banich MT, McAuley E, Harrison CR, et al. Ageing, fitness and neurocognitive function. Nature. 1999;29;400(6743):418-9.

37. Colcombe $S$, Kramer AF. Fitness effects on the cognitive function of older adults: a meta-analytic study. Psychol Sci. 2003;14(2):125-30.

38. Colcombe SJ, Kramer AF, McAuley E, Erickson KI, Scalf P. Neurocognitive aging and cardiovascular fitness: recent findings and future directions. J Mol Neurosci. 2004;24(1):9-14.

39. Themanson JR, Hillman CH. Cardiorespiratory fitness and acute aerobic exercise effects on neuroelectric and behavioral measures of action monitoring. Neuroscience. 2006;25;141(2):757-67.

40. Colcombe SJ, Kramer AF, Erickson KI, Scalf P, McAuley E, Cohen NJ, et al. Cardiovascular fitness, cortical plasticity, and aging. Proc Natl Acad Sci U S A. 2004;2;101(9):3316-21.

41. Rhyu IJ, Bytheway JA, Kohler SJ, Lange H, Lee KJ, Boklewski J, et al. Effects of aerobic exercise training on cognitive function and cortical vascularity in monkeys. Neuroscience. 2010;2;167(4):1239-48.

42. van Praag H, Kempermann G, Gage FH. Running increases cell proliferation and neurogenesis in the adult mouse dentate gyrus. Nat Neurosci. 1999;2(3):266-70.

43. van Praag H, Christie BR, Sejnowski TJ, Gage FH. Running enhances neurogenesis, learning, and long-term potentiation in mice. Proc Natl Acad Sci U S A. 1999;9;96(23):13427-31.

44. Trejo JL, Llorens-Martin MV, Torres-Aleman I. The effects of exercise on spatial learning and anxiety-like behavior are mediated by an IGF-I-dependent mechanism related to hippocampal neurogenesis. Mol Cell Neurosci. 2008;37(2):402-11.

45. Ducloux D, Motte G, Nguyen NU, Abdelfatah A, Gibey R, Chalopin JM. Homocysteine, nutritional status and insulin in renal transplant recipients. Nephrol Dial Transplant. 2002;17(9):1674-7.

46. Sachdev P. [Homocysteine and neuropsychiatric disorders]. Rev Bras Psiquiatr. 2004;26(1):50-6.

47. Arwert LI, Deijen JB, Drent ML. The relation between insulin-like growth factor I levels and cognition in healthy elderly: A meta-analysis. Growth Horm Igf Res. 2005;15(6):416-22.

48. Ramirez-Rodriguez G, Benitez-King G, Kempermann G. The new neuron formation in the adult hippocampus: Neurogenesis. Salud Ment. 2007;30(3):12-9.

49. Kerr AL, Steuer EL, Pochtarev V, Swain RA. Angiogenesis but not neurogenesis is critical for normal learning and memory acquisition. Neuroscience. 2010;24;171(1):214-26.

50. Laufs U, Werner N, Link A, Endres M, Wassmann S, Jurgens K, et al. Physical training increases endothelial progenitor cells, inhibits neointima formation, and enhances angiogenesis. Circulation. 2004;20;109(2):220-6.

51. Gavin TP, Robinson CB, Yeager RC, England JA, Nifong LW, Hickner RC. Angiogenic growth factor response to acute systemic exercise in human skeletal muscle. J Appl Physiol. 2004;96(1):19-24.

52. Van der Borght K, Kobor-Nyakas DE, Klauke K, Eggen BJ, Nyakas C, Van der Zee EA, et al. Physical exercise leads to rapid adaptations in hippocampal vasculature: temporal dynamics and relationship to cell proliferation and neurogenesis. Hippocampus. 2009;19(10):928-36.

53. Armstrong LE, VanHeest JL. The unknown mechanism of the overtraining syndrome - Clues from depression and psychoneuroimmunology. Sports Med. 2002;32(3):185-209.

54. Wong EY, Herbert J. Raised circulating corticosterone inhibits neuronal differentiation of progenitor cells in the adult hippocampus. Neuroscience. 2006;137(1):83-92.

55. Coburn-Litvak PS, Pothakos K, Tata DA, McCloskey DP, Anderson BJ. Chronic administration of corticosterone impairs spatial reference memory before spatial working memory in rats. Neurobiol Learn Mem. 2003;80(1):11-23.

56. Woodson JC, Macintosh D, Fleshner M, Diamond DM. Emotion-induced amnesia in rats: working memory-specific impairment, corticosterone-memory correlation, and fear versus arousal effects on memory. Learn Mem. 2003;10(5):326-36.

57. Oliveira VN, Bessa A, Jorge ML, Oliveira RJ, de Mello MT, De Agostini GG, et al. The effect of different training programs on antioxidant status, oxidative stress, and metabolic control in type 2 diabetes. Appl Physiol Nutr Metab. 2012;37(2):334-44.

58. Cakir-Atabek H, Demir S, PinarbaSili RD, Gunduz N. Effects of different resistance training intensity on indices of oxidative stress. J Strength Cond Res. 2010;24(9):2491-7.

59. Lin MT, Beal MF. Mitochondrial dysfunction and oxidative stress in neurodegenerative diseases. Nature. 2006;19;443(7113):787-95. 